\title{
A Case of in Utero Meconium Peritonitis in a Fetus Delivered to a Woman Diagnosed with Primary Extrahepatic Biliary Atresia
}

\author{
Maha Shahin $^{\mathrm{a}, \mathrm{b}}$, Rukhsana Iqbal ${ }^{\mathrm{a}}$, Osama Omran ${ }^{\mathrm{a}}$
}

\begin{abstract}
Fetal meconium peritonitis was detected at 20 weeks gestation in a 22-year-old primigravid woman suffering from primary extrahepatic biliary atresia. She was awaiting a liver transplant because of recurrent life threatening cholangitis when she found out that she was pregnant. On the 20 weeks detailed scan, the fetus showed severe echogenic bowel and significant ascites. There was a suspicion that the baby might have a similar condition as the mother has but this was never proved. The mother had a preterm labour at 33 weeks of gestation and she had a spontaneous vaginal delivery. The neonate was admitted to special care baby unit where she made a good progress and was discharged home 16 days later.
\end{abstract}

Keywords: Biliary atresia; Meconium peritonitis; Hyperechogenic bowel; Fetal ascitis; Intraperitoneal calcification in utero

\section{Introduction}

Meconium peritonitis (MP) is sterile chemical peritonitis resulting from intrauterine bowel perforation. It occurs in 1:35000 live births [1]. Following bowel perforation, meconium and digestive enzymes are extruded into the peritoneal cavity, inciting an intense chemical peritonitis and secondary inflammatory response. Within days, giant cells and histiocytes surround the meconium, resulting in foreign body granulomas and calcification [2].

The common underlying bowel disorders leading to MP include small bowel atresia, meconium ileus, volvulus,

\footnotetext{
Manuscript accepted for publication November 18, 2010

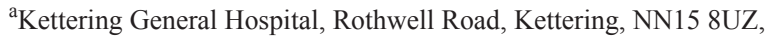
UK

${ }^{\mathrm{b}}$ Corresponding author: 10 Sanderson close, Kettering, NN15 7EP, UK. Email:mahashahin@hotmail.com

doi:10.4021/jmc94w
}

and intussusception. MP can however be idiopathic in some cases [3]. While cystic fibrosis is universally seen in cases of meconium ileus, it is only seen in $7 \%-40 \%$ of cases of MP.

A spectrum of radiographic and sonographic findings may be seen in cases of MP, depending on the underlying bowel disorder, the inflammatory response, and the interval between the perforation and the investigation. Intraperitoneal calcifications are the most common and characteristic finding on both abdominal radiographs and sonograms. They appear as highly echogenic linear or clumped foci in the abdomen, or pelvis [1]. Other findings that support the diagnosis of MP include polyhydramnios, fetal ascites, and bowel dilatation.

The prognosis depends upon the etiology. In some cases, bowel perforations may heal spontaneously and the ascites and bowel dilatation may resolve, leaving only peritoneal calcifications as the only sonographic sign of MP [4]. In other cases, the MP induced inflammation of adjacent extrahepatic biliary periductal tissue could lead to severe and protracted inflammatory reactions with accompanying fibrosis, causing secondary obliteration of the extrahepatic biliary system and biliary atresia [5].

\section{Case Report}

A 22-year-old primigravida was referred at 13 weeks gestation to book an antenatal consultant led care. The patient was known to have primary extrahepatic biliary atresia for which she has had Kasai portoenterostomy operation at 6 weeks of age. This was followed by a second laparotomy and revision of Roux loop and dissection of adhesions when she was 10 years old. She had a yearly follow-up in the paediatric liver outpatient clinic, but, in view of recurrent life-threatening cholangitis, a decision was made for liver transplant. However, she fell pregnant while she was on the waiting list for this operation.

On her booking visit, she was generally well, dating scan was normal and her liver function tests were within normal range. She had regular antenatal checks jointly with a Tertiary referral hepatic centre. At 20 weeks gestation, she was offered a detailed scan, which revealed a significant fe- 


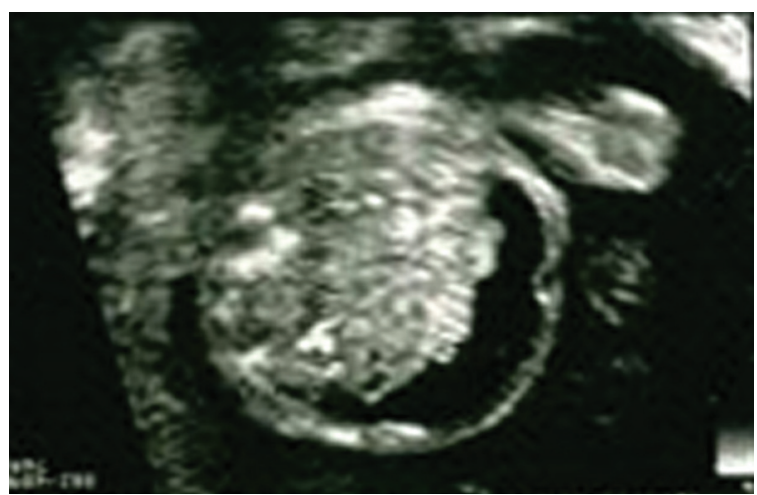

Figure 1. Ultrasound scan showing intra abdominal calcification and fetal ascitis.

tal ascites and abdominal wall oedema, marked echogenic fetal bowel and hepatic and subdiaphragmatic calcifications (Fig. 1). The echogenicity was most marked in the bowel wall than in the liver although there was no bowel dilatation. The liver appeared normal in size and vasculature and the gall bladder was clearly seen. There were no other fetal abnormalities noted and the liquor volume was within normal limits. Fetal Doppler studies were normal and uterine artery Doppler revealed a marginally raised resistance index. An infection screen was performed on the mother and it ruled out cytomegalovirus infection, toxoplasmosis, or parvovirus infection. The parents were tested for cystic fibrosis and the results were negative for common mutations. Amniocentesis at 21 weeks and five days revealed a normal fetal karyotype (46, XX). A probable diagnosis of idiopathic MP was made following exclusion of other causes of echogenic bowel. The mother was offered serial ultrasound scans and Doppler studies in a tertiary fetal medicine unit for followup of isolated ascites and hyperechogenic bowel and liver. Subsequently, during her antenatal care, the serial ultrasound scans revealed normal fetal growth and gradual subsidence of the ascites until it disappeared completely at 32 weeks. Meanwhile, the maternal liver function tests started to become increasingly deranged from 24 weeks onwards and she was managed as severe obstetric cholestasis.

The patient then presented to our maternity unit at 33 weeks in preterm labor. She received one dose of steroids and tocolytic therapy but eventually had a spontaneous vaginal delivery of a female baby weighing $1.880 \mathrm{~kg}$. The baby was born in good condition, an APGAR score of 9, 10, and 10 requiring no resuscitation. The baby was admitted to the special care baby unit due to prematurity and for further investigations.

An abdominal $\mathrm{X}$ ray showed calcification in the right side of the abdomen (Fig. 2), a chest X ray revealed streaky peri-hilar shadowing with no focal consolidation or collapse. A cranial ultrasound scan was normal. Cystic fibrosis test- ing and blood cultures were negative and routine blood tests were normal. The baby was started on IV fluid and small amounts of feed which was well tolerated with no bilious aspirates. The feeds were increased gradually and were well tolerated and the baby started to gain weight. The diagnosis was that of MP following bowel perforation with the possibility of sealing off the perforation in utero. After 16 days, the baby was considered to be in good condition to go home and a follow-up appointment was arranged in 4 months.

\section{Discussion}

Meconium peritonitis might be considered as a diagnostic possibility when hyperechogenic areas are seen within the fetal abdomen during the second and third trimesters of pregnancy. The etiology of MP is thought to be the result of a sterile chemical reaction resulting from bowel perforation in utero. A secondary inflammatory response results in the production of ascitic fluid, fibrosis, calcification, and sometimes cyst formation. The clinical manifestations and the radiological findings depend on whether the bowel perforation seals off in utero or in the neonatal period or remains patent. Antenatal ultrasound allows early detection of the condition, demonstrating free fluid, hydrocoeles, and echogenic foci representing intraperitoneal calcifications. In the newborn period, a plain abdominal radiograph demonstrating calcifications is sufficient for the diagnosis [6]. Eventually most of these disappear, as it is rare to encounter an older child with residual calcifications. Rarely, as seen in our case, MP occurs without an underlying cause, and it remains innocuous hence any intervention is not required.

Biliary atresia generally was believed to be an early developmental malformation, however there are both clinical and morphologic arguments against this early maldevelopment hypothesis [5]. Interestingly, there have been several reports in the literature suggesting that biliary atresia may

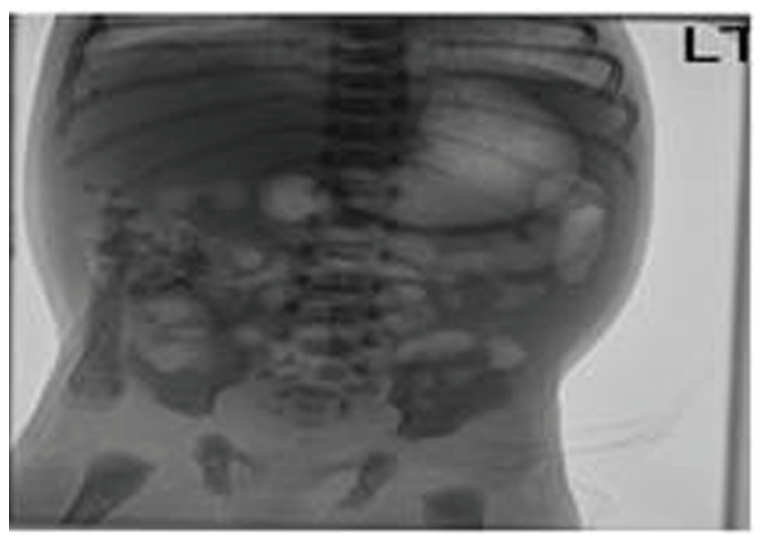

Figure 2. Abdominal $X$ ray showing calcification in the right side of the abdomen. 
be caused by MP secondary to perforation of small bowel atresia [7, 8]. So, whether the mother in our case study was originally affected by MP then later complicated by biliary atresia will remain an unanswered academic question.

\section{References}

1. Foster MA, Nyberg DA, Mahony BS, Mack LA, Marks WM, Raabe RD. Meconium peritonitis: prenatal sonographic findings and their clinical significance. Radiology 1987;165(3):661-665.

2. Forouhar F. Meconium peritonitis. Pathology, evolution, and diagnosis. Am J Clin Pathol 1982;78(2):208-213.

3. Lorimer WS, Jr., Ellis DG. Meconium peritonitis. Surgery 1966;60(2):470-475.
4. Dirkes K, Crombleholme TM, Craigo SD, Latchaw LA, Jacir NN, Harris BH, D’Alton ME. The natural history of meconium peritonitis diagnosed in utero. J Pediatr Surg 1995;30(7):979-982.

5. Han SJ, Han A, Choi SH, Oh JT, Hwang EH. Biliary atresia associated with meconium peritonitis caused by perforation of small bowel atresia. J Pediatr Surg 2001;36(9):1390-1393.

6. Reynolds E, Douglass B, Bleacher J. Meconium peritonitis. J Perinatol 2000;20(3):193-195.

7. Kishida Y., Ito T., Nagaya M., et al. Three cases of biliary atresia associated with small bowel atresia.Ann Rep Nagoya Uni Br Hosp 1988; 22:17-23.

8. Chung MY, Ko TY, Huang CB, Lee CH, Hsieh CS. Biliary atresia associated with meconium peritonitis. Chang Gung Med J 2006;29(2):203-206. 